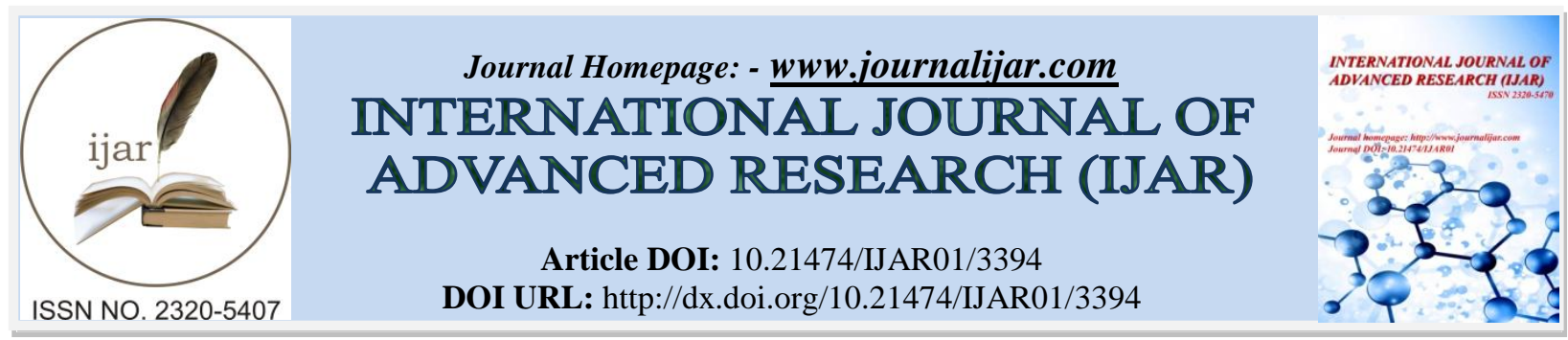

RESEARCH ARTICLE

\title{
TOTAL KNEE ARTHROPLASTY IN OSTEOARTHRITIC PATIENTS WITH SEVER VARUS DEFORMITY.
}

\author{
Alaa Mostafa El-ngehy ${ }^{1}$, Mahmoud Ahmed El-sebaie ${ }^{2}$, Mahmoud Mohamed Matter ${ }^{1}$ and Ahmed Mohamed \\ Sallam ${ }^{3}$. \\ 1. Professor of orthopedic surgery, Faculty of Medicine, Zagazig University, Egypt. \\ 2. Professor of orthopedic surgery, Faculty of Medicine, Ain Shams University, Egypt. \\ 3. Assistant lecturer of orthopedic surgery, Faculty of Medicine, Zagazig University, Egypt.
}

\section{Manuscript Info}

(.........................

Manuscript History

Received: 19 December 2016

Final Accepted: 18 January 2017

Published: February 2017

Key words:-

Total Knee Arthroplasty, osteoarthritis, varus.

\section{Abstract}

Objective:The aim of this study is to evaluate the results of knee arthroplasty in cases of osteoarthritis with sever varus deformity.

Subjects and Methods:This study is prospective study was conducted involving 20 patients who underwent primary posterior stabilized total knee arthroplasty due to osteoarthritis with sever varus deformity.

Results:Using Knee Society Scoring(KSC) system reveals that, the average post-operative score was 94.3 (range from 80 to97) compared with average pre-operative score 17.5 (range from 0 to 22).

Conclusion:Total knee arthroplasty in osteoarthritic patients with severvarus deformity gives the best solution in managing pain,deformity, improve walking and improve range of motion.

Copy Right, IJAR, 2017,. All rights reserved.

\section{Introduction:-}

Knee replacement surgery is one of the most successful surgeries in orthopedics. Hundreds of thousands of these operations are now carried out every year worldwide with excellent results. Knee replacement becomes necessary when the knee joint has been damaged from any cause and the resulting pain cannot be satisfactorily be controlled by other means. The usualproblem that can end up in the need for total knee replacement is chronic arthritis (Sandesh et al., 2015).

Varus deformity is predominantly the commonest deformity in candidates for total knee arthroplasty. This deformity possibly underlies pathomechanics that led to the progression of the arthritic process, and may compromise the outcome of the arthroplasty itself. Obtaining a well positioned and stable prosthetic construct with restoration of the normal mechanical axes of the limb and joint line have been shown to have an important bearing on the final outcome of knee replacement operations(Elsebaei, 2001)

Varus deformity is defined by any preoperativetibiofemoral angle less than naturally occurring anatomic valgus(Laskin, 1996).

\section{Patient And Methods:-}

This study was carried out in Orthopedic Surgery Departments, Faculty of Medicine, Zagazig and Ain Shams Universityin the period between April 2014 and October 2016, a prospective study was conducted involving 15 patients with 20 knees who underwent primary total knee arthroplasty due to severvarus osteoarthritis. 
We included patients with primary degenerative varus osteoarthritis with tibio-femoral angle more than 15 degrees(more than 20 degrees varusmalalignment)with average preoperative tibio-femoral angle of 26.2 degrees (range from 22 to 41 varus), aged above 50 yearswith the mean age at time of surgery is 65.4 (ranging from 58 to 74 years)and their deformities are totally articular i.e. wear and erosion of the medial compartment of the joint.

The group of patients included 1 male (7\%) and 14 females (93\%).Five patients had the right knee replaced, five patients had the left one, while five patients had bilateral total knee replacement 2 of them were done bilateral simultaneous at the same setting. All patients had flexion knee deformity. Fifteen (75\%) knees had mild flexion deformity (less than $\left.15^{\circ}\right)$ while five $(25 \%)$ knees had moderate flexion deformity $\left(15^{\circ}-30^{\circ}\right)$.

The diagnosis was primary varus osteoarthritis in all the cases (100\%) and any patient with knee arthritisrather than primary severvarus osteoarthritis with deformity more than 20 degrees varusmalalignment, inflammatory arthritis (e.g. Rheumatoid arthritis),post traumatic O.A. knee, extra-articular deformity, and other general contraindications of arthroplasty was excluded.

Pre-operative clinical,laboratory and radiolodical assessment of the patients was done. Knee Society Scoring (KSS) systemwas taken to each patient pre and post-operative. Follow up for two years was done.

Prophylaxis against D.V.T., proper antibiotic coverage must be taken in consideration.

\section{Operative Techniqe:-}

In our stude 13 patients was given epidural anesthesia, 2patients spinal anesthesia. Tourniquet was applied in all patients.

Midline skin incision was used with avoidance of skin undermining(Bellemans et al, 2008).

The main approach which was performed in the present study was the medial para-patellar approach in seventeen knees $(85 \%)$. While three knees $(15 \%)$ were done by the subvastus approach.

We used the sequential medial release, after initial dissection of the soft tissues along the medial joint line, including the deep medial collateral ligament, osteophytes were removed from the medial distal femur and proximal tibia.

The distal femur was cut at $5-7^{\circ}$ valgus to the femoral shaft axis using intramedullary instrumentation (perpendicular to the mechanical axis of the femur. Like the femoral resection, resection of the proximal tibial surface is based on the height of the intact lateral bone surface. A maximum thickness of 8-10 mm cut is removed from the medial tibial plateau perpendicular to the mechanical axis of the tibia, which often leaves a large bony defect on the medial side of the tibia that was reconstructed either by bone graft or metal augmentation with or without use of long stem tibial component.

Metal augments were done in uncontained (steeply defects) defects more than $10 \mathrm{~mm}$ (done in 7 cases). They are either $1 / 2$ wedges ( 3 cases) or $1 / 2$ blocks (4cases) (according to the geometry of the defect).In cases of complex reconstruction of tibialdefects, long stem tibial component was used to unload the deficient metaphyseal bone (12 cases).

Bone grafts was used in reconstruction of contained defects usually without fixation (done in 3 cases). Or uncontained defects 5-10 $\mathrm{mm}$ using special technique for its fixation (done in 3 cases). 

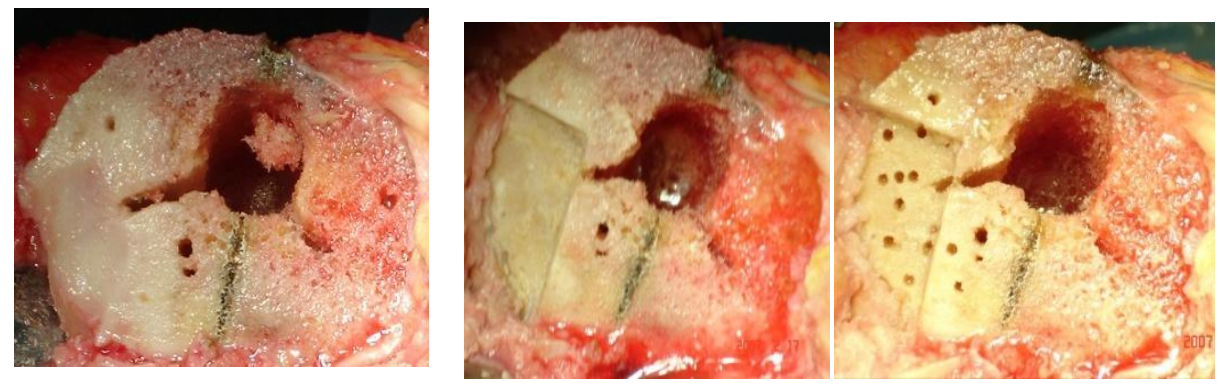

$-1-$
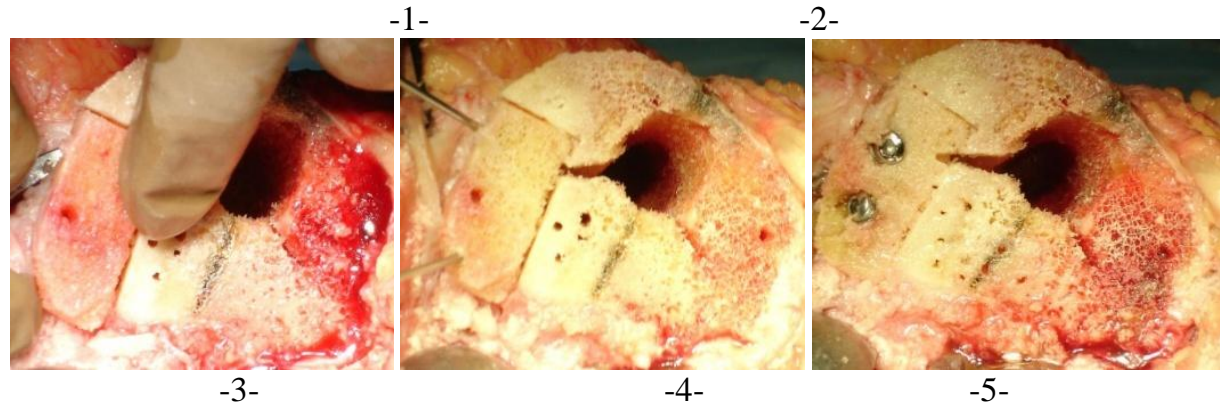

Technique of bone grafting in management of the tibialdefect.

\section{Results:-}

Clinical results:-

\section{Knee Society Score:-}

At last follow up, the average Knee Society Score (KSS) was 94.3 (range from 80 to 97) compared with average pre-operative KSS of 17.5 (range from 0 to 22).Seventeen (85\%) knees had excellent results (85 to 100 points), three (15\%) knees had good results (70 to 84 points), No knees had fair results (range 60 to 69 points) or poor results ( $<60$ points).

\section{Knee Function Score:-}

At the last follow up, the average Knee Function Score(KFS) was 88.5 (range from 83 to 94) compared with average preoperative knee function score of 17.8 (range from zero to 26).Three (15\%) knees had excellent results ( 85 to 100 points), twelve (60\%) knees had good results (70 to 84 points) and four knees (13.33\%), two (10\%) knees had fair results (range 60 to 69 points) and one (5\%) knee had poor result (less than 60).

\section{Post-operative range of motion:-}

At last follow up, the average post-operative range of motion was $100.4^{\circ}$ (range from $0-120^{\circ}$ ). There was one knee (5\%) with maximum range of above 110,eighteen knees (90\%)flexion between 80 and 110 degrees, and one knee (5\%) with range below 80 degrees. In comparison to the pre-operative range of motion the average was $87.3^{\circ}$. Fifteen knees (75\%) were in the range between 80-110 degrees, zero knees with range above 110 degrees and 5 knees $(25 \%)$ were below range of motion of 80 degrees.

\section{Pain score:-}

At last follow up, fifteen knees out of twenty (75\%) had no pain (score 50). Five knees (25\%) had very mild or occasional pain (score 45), No other knees in the study had pain on stairs or walking.

\section{Radiological results:-}

Limb alignment (Tibio-femoral Angle):-

The average post-operative tibio-femoral valgus angle was 6.8 degrees (range from 4 to 11 valgus), compared with average pre-operative tibio-femoral angle of 26.2 degrees (range from 22 to 41 varus). Eighteen knees (90\%) had a valgus angle within 5-10 degrees, while one knee (5\%) had excessive valgus angle of more than 10 degrees and one knee $(5 \%)$ had valgus angle less than 5 degrees. 


\section{Complication:-}

In this study,three knees had complications (15\%).

Complications and its ratio:-

\begin{tabular}{|c|c|c|c|}
\hline Complication & Case No. & Number & $\%$ \\
\hline Superficial skin infection & 5 & 1 & $5 \%$ \\
\hline Partial patellar tendon rupture & 12 & 1 & $5 \%$ \\
\hline Fissure fracture of med. tibial condyle & 2 & 1 & $5 \%$ \\
\hline
\end{tabular}
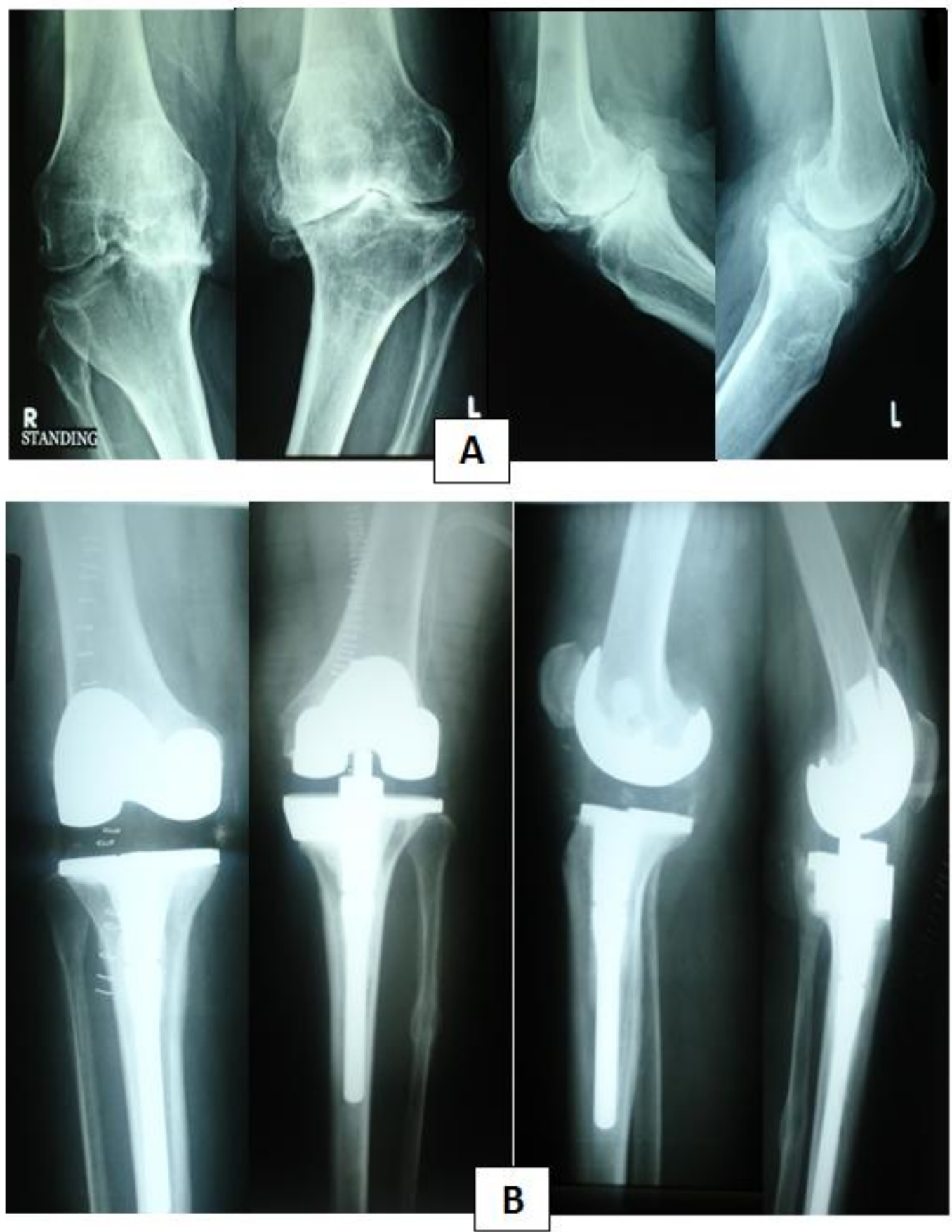

AP \& Lat. views of both knees: A: pre-operative B: immediate post-operative. 


\section{Discussion:-}

Varus deformity is predominantly the commonest deformity in candidates for total knee arthroplasty. Obtaining a well positioned and stable prosthetic construct with restoration of the normal mechanical axes of the limb and joint line have been shown to have an important bearing on the final outcome of knee replacement operations.

At last follow up, the average Knee Society Score (KSS) was 94.3 (range from 80 to 97) compared with average pre-operative KSS of 17.5 (range from 0 to 22).

Sandesh et al,(2015)reported in their study improvement of the mean preoperative knee society score i.e. knee and function scores were 29.45(range:15-52) and 26.50(range: 10-40) respectively to 84(range: 60-92) and 79.5(range: 70-90) respectively.Their results were coincided with the present study results.

Mullaji et al,(2005)reported in their series improvement of the mean knee society score from 22.8 preoperatively to 99.1 postoperatively and the function knee score from 22.8 to 72.1 . Their results were coincided with the present study results.

Dixon et al,(2004)also reprted that the KSS increased from a mean of 24 (range from 0 to 43) to a mean of 94 (range from 78 to 100). The FSimproved from a mean of 34 (range, 0 to 70 ) to amean of 85 (range, 45 to 100).Withtheir results were highlycoincided with the present study results.

Merrill et al, (2004)reported improvement ofthe preoperative knee score for the severe deformity from 44.5 to 87.8 with significant improvement.

Tenny et al(1991)reported that in their study the mean post-operative knee society score was 89 . Sixteen knees $(59 \%)$ in the varus deformity group were rated excellent and 11 knees $(41 \%)$ good. There were no fair or poor results. Their results were also go hand in hand with the results of the current study.

There is marked improvement of deformity either in coronal plane or in sagittal plane.The average post-operative tibio-femoral valgus angle was 6.8 degrees (range from 4 to 11 valgus), compared with average pre-operative tibiofemoral angle of 26.2 degrees (range from 22 to 41 varus). Eighteen knees (90\%) had a valgus angle within 5-10 degrees, while one knee (5\%) had excessive valgus angle of more than 10 degrees and one knee $(5 \%)$ had valgus angle less than 5 degrees.

There is marked improvement in pain sensation either at rest or during work.Improvement of walking distance postoperatively occurs.

\section{Conclusion:-}

Proper pre-operative evaluation of the patients clinically and roentgenographicaly is mandatory.

There was no statistically difference in the mean KSS and FKSS post operatively between age groups.

Epidural or Spinal anesthesias(unless contraindicated) are the better options to avoid the risk of general anesthesia and maximize the period of analgesia, so faster regain of ROM postoperatively.

Subvastus approach is better than medial para-patellar approach as it gives the chance for early post-operative rehabilitation as well as it minimize patellar complication. On the other hand, it is better to be avoided in obese patients with short patellar tendon and also in patients with severe fixed varus deformity especially is accompanied by flexion deformity.

Referencing distal femoral resection and proximal tibial cut (measured resection technique) from the intact lateral surfaces has the advantage of restoration of the normal joint line, preserving bone stock for future revisions and avoiding the increase in lateral laxity.

Sequential medial soft tissue release must be performed to provide adequate stability without over releasing. PCL must be released in severe fixed varus deformity especially if accompanied by flexion deformity. 
Patients with severe degrees of varus $\mathrm{OA}$ are more likely to have medial tibial bone defects and must be reconstructed by either bone grafts or metal augments and in the same time uploading the tibial surface by long stem to distribute about $1 / 3$ of the load stresses to the diaphysis.

Meticulous closure of the arthrotomy and preservation of part of the medial meniscus remnant attached to the medial capsular sleeve for adequate closure of the medial structures, proper determination of the joint level for future revisions.

Patients with mild degrees of flexion deformity show much improvement in the KSS compared to those with severe degrees of flexion deformity.

Locked long tibial stems could be used in managing concomitant tibial fractures.

Postoperative maintance of full extension is the first priority. Strengthening exercise, and increase range of movement is started as rapid as possible. In non complicated cases, walking using a walker from second day postoperative can be started. Walker must be used for one month. Proper anticoagulant, antibiotic, and analgesics must be given to patient.

\section{References:-}

1. Sandesh Reddy Y.,Jambu N. and Samuel B. (2015):Functional and radiological outcome of total knee replacement in varus deformity of the knee.Int.J.Curr.Microbiol.App.Sci 4(4): 934-938

2. Mahmoud Elsebai (2001): Management of different forms of varus deformity during total knee arthroplasty. Pan Arab J. Orth. Trauma. Vol.(5) No. (1)

3. Laskin RS (1996): Total knee replacement with posterior cruciate ligament retention in patients with fixed varus deformity. ClinOrthopRelat Res. 331; p 29

4. Bellemans J, Vandenneucker H, Vanlauwe J (2008): Total knee arthroplasty in the valgus knee using a standard medial approach: Interact surgery;3, pp:2-5, springer

5. Mullaji AB, Vinod P, Gaurav J (2005):Total Knee Arthroplasty for Profound Varus deformity. The Jornal of Arthroplasty, 20(5): 550-561

6. Michael C. Dixon, Dominik Parsch,Richard R. Brown, and Richard D. Scott (2004): The Correction of Severe Varus Deformity in Total Knee Arthroplasty by Tibial Component Downsizing and Resection of Uncapped Proximal Medial BoneThe Journal of Arthroplasty Vol. 19 No. 1

7. Merrill A. Ritter, MD, Gregory W. Faris, BS, Philip M. Faris, MD, and Kenneth E. Davis, MS (2004): Total Knee Arthroplasty in Patients With AngularVarus or Valgus Deformities of $>20^{\circ}$. The Journal of Arthroplasty Vol. 19 No. 7

8. Teeny SM, Krackow KA, Hungerford DS, et al (1991): Primary total knee arthroplasty in patients with severe varus deformity. ClinOrthopRelat Res. 273; p 19 